\title{
Découverte d'une infection à VIH à partir d'une sialomégalie bilatérale
}

\author{
Marc Baranes ${ }^{1,2,3, \star}$, Alp Alantar ${ }^{1}$, Louis Maman ${ }^{2,3}$, Catherine Sequert ${ }^{4}$, \\ Jean-Marc Galéazzi ${ }^{1}$ \\ 1 Service d'Odontologie, Hôpital Max Fourestier, 92000 Nanterre, France \\ 2 Service d'Odontologie, Hôpital Charles Foix, 94200 Ivry-sur-Seine, France \\ 3 Faculté de Chirurgie dentaire, 1 rue Maurice Arnoux, 92120 Montrouge, France \\ 4 Service d'ORL, Hôpital Max Fourestier, 92000 Nanterre, France
}

(Reçu le 11 novembre 2009, accepté le 28 novembre 2009)

Mots clés : glandes salivaires / VIH / hyperplasie lymphoïde kystique

Key words: salivary glands / HIV / cystic lymphoid hyperplasia

\begin{abstract}
Résumé - L'atteinte des glandes salivaires chez les patients infectés par le virus de l'immunodéficience humaine (VIH) est fréquente. On distingue des pathologies infectieuses, des néoplasies et l'hyperplasie lymphoïde kystique (HLK). Face à une sialomégalie persistante, l'hypothèse d'une HLK liée à l'infection par le VIH doit être évoquée. Le tissu lymphoïde des glandes salivaires représente une cible pour le VIH et son effet cytopathogène est directement à l'origine du développement de cette lésion. L'observation présentée est celle d'une patiente ayant une sialomégalie bilatérale. La démarche diagnostique a conduit à la découverte de sa séropostivité pour le VIH.
\end{abstract}

Abstract - Discovery of HIV infection from a bilateral sialomegalia. Disease of salivary glands in patients infected by Human Immunodeficiency Virus (HIV) is frequent. It includes infectious disease, neoplasia and cystic lymphoid hyperplasia. In front of a persistant salivary gland swelling, the hypothesis of lymphoepithelial cysts correlated with HIV infection must be reminded. Lymphoid tissue of salivary gland is a target for HIV and his cytopathogenesis is directly the source of the lesion's growth. The case reported is about a patient with bilateral salivary glands swelling. The diagnostic approach leads to the discovery of a HIV seropositivity.
Les manifestations bucco-faciales associées à l'infection VIH ont été décrites avec l'observation des premiers cas dans les années 1980. Les plus fréquentes sont les candidoses oropharyngées, les gingivites et les parodontites ulcéronécrotiques, le sarcome de Kaposi, l'infection herpétique, les verrues, les condylomes acuminés, les aphtoses récidivantes et les lymphomes. L'atteinte des glandes salivaires est moins fréquente mais elle peut être le signe évocateur d'une infection à VIH. Cette observation clinique est celle d'une patiente présentant une tuméfaction importante des glandes parotides et submandibulaires. La démarche diagnostique a orienté vers une hyperplasie lymphoïde kystique (HLK) liée à l'infection par le VIH; l'HLK étant le seul signe clinique évocateur chez cette patiente. L'atteinte salivaire chez les patients infectés par le VIH se traduit principalement par les sialadénites chroniques et les sialoses infectieuses virales (EBV, CMV) ou

\footnotetext{
*Correspondance : marcbaranes@gmail.com
}

bactériennes. Ces affections sont indirectement liées au VIH car secondaires à l'immunodépression induite. L'HLK est plus rare mais elle est directement liée à l'effet cytotoxique du VIH.

\section{Observation}

Une patiente originaire de Côte d'Ivoire, âgée de 40 ans, est adressée par son chirurgien dentiste pour l'extraction en urgence de la 47, responsable de vives douleurs. L'entretien médical ne retient aucun antécédent général ni traitement en cours. La patiente se plaint depuis trois jours de douleurs mandibulaires droites qui ne cèdent pas à la prise de paracétamol. L'examen exobuccal met en évidence la présence de tuméfactions bilatérales des régions parotidiennes et submandibulaires, plus marquées du côté droit (Fig. 1). Ces tuméfactions, asymptomatiques et souples à la palpation, sont 

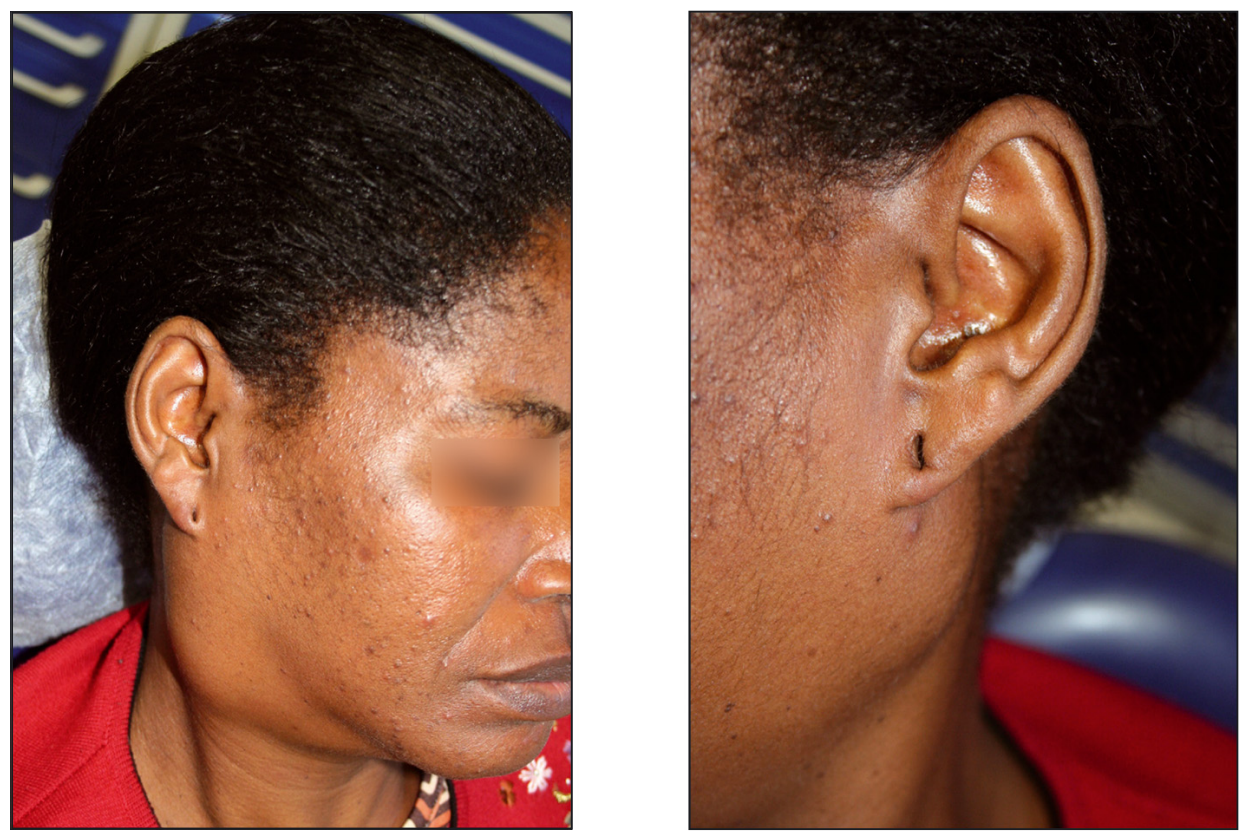

Fig. 1. Tuméfaction bilatérale des régions parotidiennes et submandibulaires et volumineuse adénopathie sous-digastrique droite. Fig. 1. Bilateral swelling of parotid and submandibular areas and big right susdigastric adenopathy.

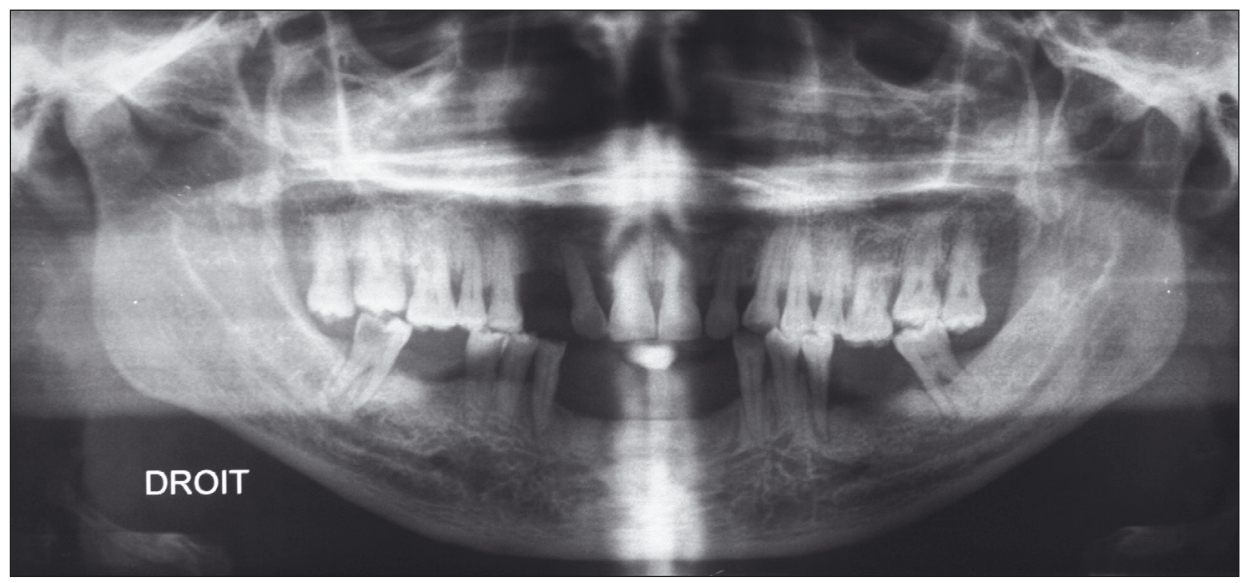

Fig. 2. Radiographie panoramique montrant une carie perforante et une image radioclaire périapicale sur la 47. Fig. 2. Panoramic radiography shows a deep decay and periapical raidolucency on 47.

présentes depuis trois mois. L'ouverture buccale est normale. L'examen endobuccal montre la présence d'un édentement maxillaire et mandibulaire non compensé ainsi qu'un délabrement coronaire important sur la 47. Le test au froid sur cette dernière est négatif et la percussion douloureuse. La radiographie panoramique (Fig. 2) confirme l'atteinte pulpaire sur 47 et la présence d'une image radioclaire péri-apicale. Le diagnostic d'abcès péri-apical aigu est posé et un traitement de première intention prescrit : antibiothérapie à large spectre (amoxicilline $2 \mathrm{~g} . \mathrm{j}^{-1}$ associée au métronidazole 1,5 g.j $\mathrm{j}^{-1}$ pendant 6 jours) et des antalgiques (paracétamol codéiné) ; l'extraction de la 47 étant programmée dans un second temps. La patiente apporte alors une échographie cervicofaciale réalisée un mois auparavant, prescrite par son médecin généraliste. Elle met en évidence « de multiples adénopathies bilatérales mesurant au maximum 40 à $50 \mathrm{~mm}$ sur leur plus grand axe » et « une hypertrophie des glandes sous-maxillaires et parotidiennes qui présentent un aspect hétérogène avec plusieurs petites formations kystiques infracentimétriques » (Fig. 3).

Face à la confirmation d'une sialomégalie bilatérale, plusieurs hypothèses diagnostiques peuvent être alors évoquées :

- sialomégalies infectieuses : infection d'origine lithiasique, infection virale (oreillons, CMV...), infection bactérienne (tuberculose...);

- pathologies tumorales : adénome pléomorphe, cystadénolymphome, carcinome adénoïde kystique, lymphome... 

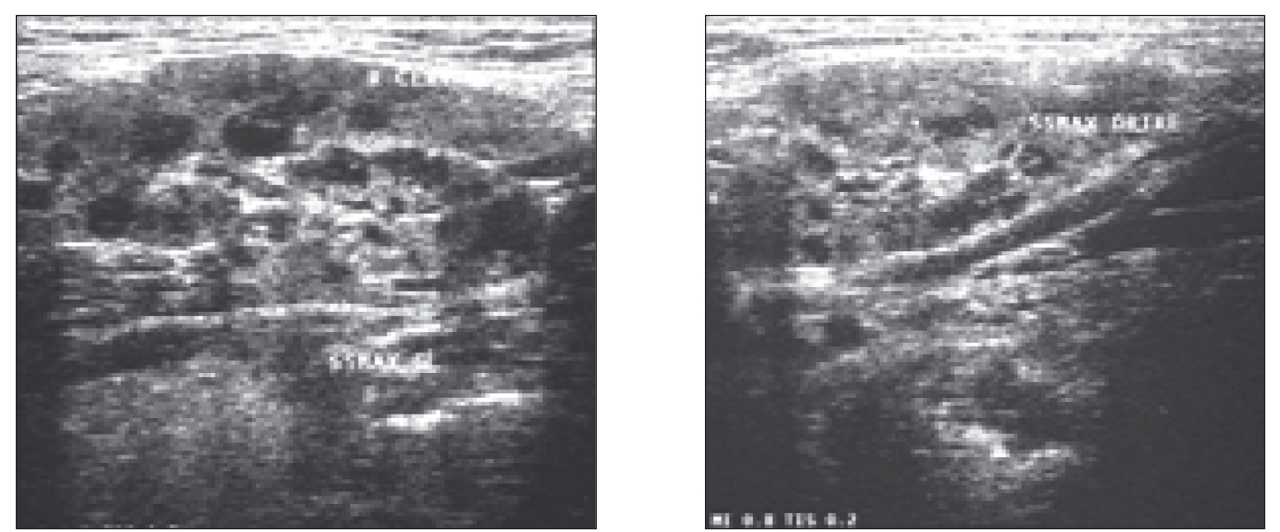

Fig. 3. Echographie des glandes salivaires de profil : formations kystiques intra-glandulaires dans les glandes submandibulaires droite et gauche.

Fig. 3. Salivary's glands sonography: intraglandular cysts in left and right submandibular glands.

- sialadénoses (dystrophies salivaires) : anorexie/boulimie, excès d'aliment riche en amidon (pain, pomme de terre) ;

- sialoses systémiques : syndrome de Gougerot-Sjögren, sarcoïdose, VIH...

Face à la symptomatologie discrète et au caractère bilatéral, les étiologies infectieuses et les sialadénoses sont écartées. Afin d'affiner la démarche diagnostique, une tomodensitométrie cervico-faciale est prescrite. Elle met en évidence une hypertrophie des glandes parotides et submandibulaires avec de multiples images hypodenses intraglandulaires ainsi qu'une polyadénopathie cervico-faciale (Fig. 4). Le diagnostic s'oriente alors vers une HLK associée à un syndrome lymphadénopathique en relation avec l'infection par le VIH. Un bilan biologique et une sérologie VIH sont réalisés. La patiente ne vient pas à ses rendez-vous de contrôle malgré plusieurs relances téléphoniques. Quelques jours plus tard, elle se présente au service des urgences de l'hôpital pour des douleurs thoraciques importantes avec une forte toux et une hyperthermie $\left(38,2^{\circ} \mathrm{C}\right)$. Un bilan biologique est réalisé montrant une hyperleucocytose à polynucléaires neutrophiles et un bilan inflammatoire altéré (CRP à $274 \mathrm{mg} . \mathrm{L}^{-1}$ ). La radiographie pulmonaire met en évidence une opacité basale gauche (Fig. 5) et le diagnostic de pleuropneumopathie basale gauche infectieuse est posé. La patiente est hospitalisée dans le service de pneumologie où on lui administre une antibiothérapie (amoxicilline $3 \mathrm{~g} . \mathrm{j}^{-1}$ ). La sérologie confirme la séropositivité pour le VIH1. La pleuropneumopathie régresse rapidement et une prise en charge en médecine interne est programmée pour proposer une stratégie thérapeutique antivirale.

\section{Commentaires}

La fréquence des affections des glandes salivaires chez les patients infectés par le VIH est élevée; elle est estimée à environ $50 \%$ de la population séropositive pour le VIH [1]. La plupart de ces affections sont asymptomatiques et passent souvent inaperçues. Il existe de nombreuses étiologies pour cette atteinte salivaire : infections bactériennes, virales et fongiques, lymphomes, tumeurs parotidiennes, HLK. . .
Le terme de kyste lympho-épithélial a été introduit par Bernier et Bhaskar en 1958 [2]. Ils ont d'emblée exclu l'origine embryologique de cette lésion et proposé l'hypothèse du développement de ces kystes à partir des ganglions lymphatiques intraparotidiens. Ces kystes ont été décrits initialement dans le syndrome de Gougerot-Sjögren dans les années 1950. Le lien avec l'infection à VIH a été établi dans les années 1980, on parle alors d'HLK $[3,4]$. L'incidence de l'HLK est estimée à environ $5 \%$ de la population séropositive pour le VIH $[1,5]$. Elle atteint le plus souvent les patients dans les phases précoces de l'infection. Cependant quelques cas ont été observés à des stades avancés de la maladie $[1,6]$. L'atteinte parotidienne est la plus fréquente (90\% des cas), elle est uni- ou bilatérale, associée à une lymphadénopathie périphérique diffuse. L'atteinte des glandes submandibulaires et sublinguales peut être aussi observée; elle est le plus souvent associée à une atteinte parotidienne.

L'examen clinique retrouve la présence de tuméfactions importantes des régions parotidiennes et parfois submandibulaires, uni- ou bilatérales, de consistance souple, et indolores à la palpation. Les tuméfactions se développent en quelques semaines, voire en quelques mois, parfois associées à un syndrome sec.

L'examen tomodensitométrique met en évidence une hypertrophie des glandes salivaires et un aspect polykystique : multiples images hypodenses, arrondies, intraglandulaires [7]. Le diagnostic peut être confirmé par la cytoponction à l'aiguille fine, mais la spécificité de cette technique a ses limites et le résultat doit être confronté à la clinique $[8,9]$.

L'examen histologique de l'HLK montre des cavités kystiques bordées par un épithélium de type malpighien, sans kératinisation de surface, au sein d'un tissu lymphoïde hyperplasique [10]. L'épithélium est le siège d'une importante exocytose composée de lymphocytes, parfois retrouvés dans le liquide des cavités kystiques. L'HLK représente un important réservoir de VIH, confirmé par la présence d'une grande quantité de p24 et d'ARN viral, et d'une forte activité de réplication du VIH dans les cellules dendritiques des nodules lymphoïdes 

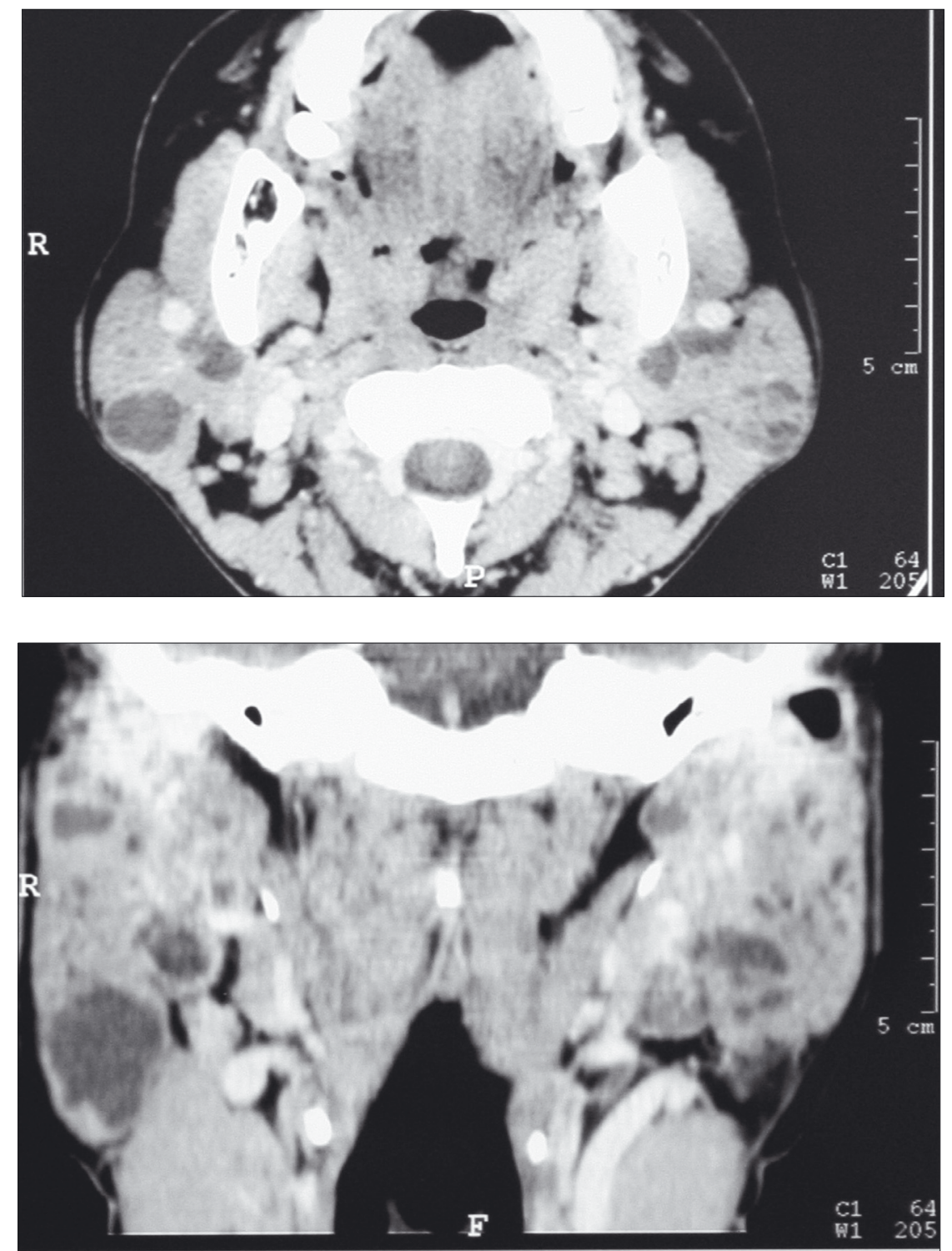

Fig. 4. Tomodensitométrie cervico-faciale : augmentation de volume des glandes salivaires avec de multiples images hypodenses intraglandulaires, et polyadénopathie cervico-faciale.

Fig. 4. Cervicofacial tomodensitometry: enlargement of salivary glands with many hypodensity areas and cervicofacial polyadenopathy.

intercavitaires $[10,11]$. L'HLK est directement lié à l'effet cytopathogène du VIH.

La pathogenèse de l'HLK reste incertaine. Deux hypothèses s'affrontent. La première considère que l'HLK prend naissance et se développe dans les ganglions lympho-épithéliaux intraglandulaires $[3,12,13]$. L'autre considère que l'HLK prend naissance dans l'infiltration lymphocytaire du tissu glandulaire et correspond à l'évolution sur un mode kystique d'une variété de sialadénite auto-immune $[14,15]$. Le cystadénolymphome a un aspect morphologique très proche mais il est rencontré dans un contexte clinique différent : c'est une lésion unique chez un sujet masculin de plus de 50 ans, séronégatif pour le VIH.
L'évolution de l'HLK est en général bénigne et une régression spontanée est fréquente avec la mise en place de la trithérapie [16]. Cependant quelques rares cas de transformation maligne en lymphome hodgkinien ont été rapportés $[16,17]$. Une surveillance régulière est nécessaire mais aucune intervention n'est indiquée face au caractère totalement bénin de cette affection. Le recours à une exérèse chirurgicale n'est envisagé que dans les rares cas associés à une gêne esthétique et fonctionnelle importante, qui ne régressent pas sous trithérapie ; d'autres traitements peuvent être proposés : sclérothérapie aux tétracyclines [18] ou radiothérapie [19] mais ces traitements paraissent disproportionnés pour une lésion le plus souvent bénigne. 


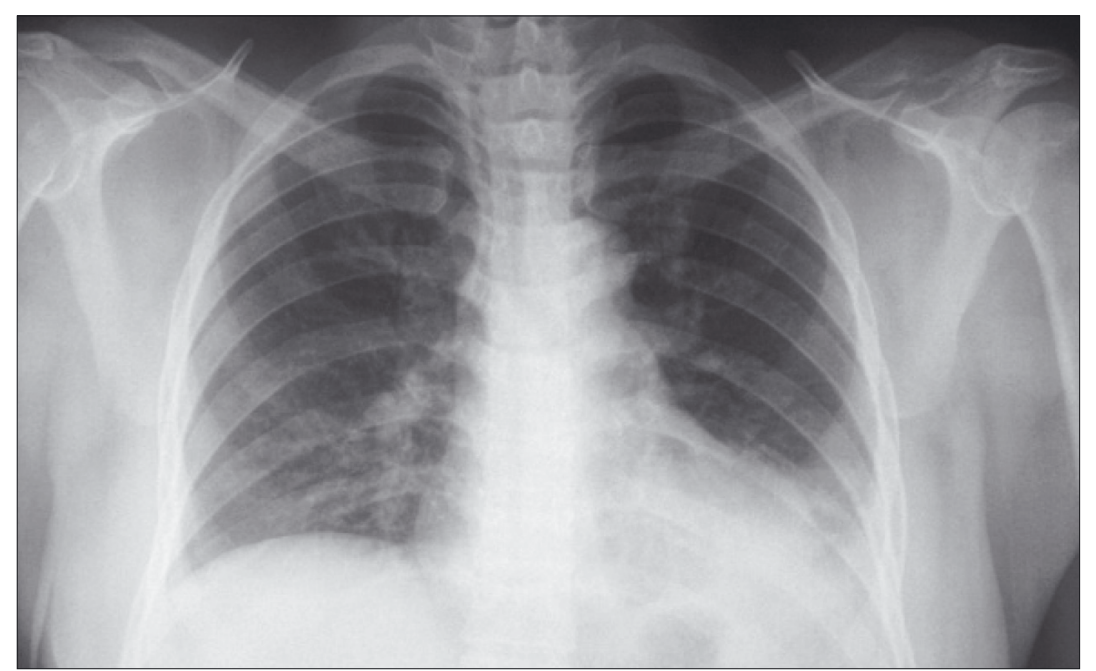

Fig. 5. Radiographie pulmonaire : radio-opacité basale gauche.

Fig. 5. Chest X-ray: left basal radio-opacity.

\section{Références}

1. Vargas PA, Mauad T, Bohm GM, Saldiva PH, Almeida OP. Parotid gland involvement in advanced AIDS. Oral Dis 2003;9:55-61.

2. Bernier JL, Bhaskar SN. Lymphoepithelial lesions of the salivary glands. Histogenesis and classification based on 186 cases. Cancer 1958;11:1156-79.

3. Ryan JR, Ioachim HL, Marmer J, Loubeau JM. Acquired immunodeficiency syndrome-related lymphoadenopathies presenting in the salivary glands lymph nodes. Arch Otolaryngol 1985;111:554-6.

4. Shaha AR, DiMaio T, Webber C, Thelmo W, Jaffe BM. Benign lymphoepithelial lésions of the parotid. Am J Surg 1993;166:403-6.

5. Allen EA, Ali SZ, Mathew S. Lymphoid lesions of the parotid. Diagn Cytopathol 1999;21:170-3.

6. Fortuno-Mar A, Mayayo E, Castillo A, Guiral H. A lymphoepithelial cyst of the parotid gland in an advanced stage of HIV infection. A rare association. An Otorrinlaringol Ibero Am 1999;26:469-75.

7. Barry B, Baujat B, Guedon C, Toublanc M, Depondt J, Gehanno P. Hyperplasie lymphoépithéliale de la parotide et infection par le VIH. Ann Oto-laryngol Chir Cervicofac 1995;112:387-92.

8. Stewart CJ, MacKenzie K, McGarry GW, Mowat A. Fine-needle aspiration cytology of salivary gland: a review of 341 cases. Diagn Cytopathol 2000;22:139-46.

9. Schindler S, Nayar R, Dutra J, Bedrossian CW. Diagnostic challenges in aspiration cytology of the salivary glands. Semin Diagn Pathol 2001;18:124-46.

10. Hofman P. Aspects histopathologiques de l'infection par le VIH. Rev Francoph Lab 2007;388:49-54.
11. Labouyrie E, Merlio JP, Beylot-Barry M, Delord B, Vergier B, Brossard G, Lacoste D, Beylot J, Leng B, Fleury H. Human immunodeficiency virus type 1 replication within cystic lymphoepithelial lésion of the salivary gland. Am J Clin Pathol 1993;100:41-6.

12. Ioachim HL, Ryan JR. Salivary gland lymphadenopathies associated with AIDS. Hum Pathol 1988;19:616-9.

13. Elliott JN, Oerte YC. Lymphoepithelial cysts of the salivary glands. Histologic and cytologic features. Am J Clin Pathol 1990;93:39-43.

14. Ulirsch RC, Jaffe ES. Sjögren's syndrome-like illness associated with the acquired immunodeficiency syndrome-related complex. Hum Pathol 1987;18:1063-8.

15. Maiorano E, Favia G, Viale G. Lymphoepithelial cysts of salivary glands: an immunohistochemical study of HIV-related and HIVunrelated lésions. Hum Pathol 1998;29:260-5.

16. Mandel L, Surattanont F. Bilateral parotid swelling: A review. Oral Surg Oral Med Oral Pathol Oral Radiol Endod 2002;93:221-37.

17. Del Bono V, Pretolesi F, Pontali E. Possible malignant transformation of benign lymphoepithelial parotid lesions in human immunodeficiency virus-infected patients: report of three cases. Clin Infect Dis 2000;30:947-9.

18. Lustig LR, Lee KC, Murr A, Deschler D, Kingdom T. Doxycycline sclerosis of benign lymphoepithelial cysts in patients infected with HIV. Laryngoscope 1998;108:1199-205.

19. Kooper DP, Leemans CR, Hulshof MC, Claessen FA, Snow GB. Management of benign lymphoepithelial lesions of the parotid gland in human immunodeficiency virus-positive patients. Eur Arch Otorhinolaryngol 1998;255:427-9. 